\title{
Patrón de respuesta aberrante: EVIDENCIAS EN LA PRECISIÓN PSICOMÉTRICA ${ }^{\star}$

\author{
Manuel Escobar Alba \\ Mónica Gamio Tupia \\ Carlos Malacas Bautista ${ }^{\star}$ \\ César Merino Soto ${ }^{\star \star \star}$
}

Palabras clave: validez; respuesta; evaluación.

Una de las herramientas importantes para los psicólogos son los test psicológicos, los cuales nos permiten hacer inferencias sobre la cantidad de un atributo en un individuo. Pero, específicamente, el puntaje es el medio que representa el nivel del atributo que posee. Para una apropiada representación e interpretación de un puntaje, se asume teóricamente una relación funcional entre el puntaje y el atributo latente; esta relación puede ser lineal o de otro tipo. Sin embargo, es frecuente hallar que esta relación es alterada por otras fuentes diferentes que contribuyen a invalidar la interpretación del puntaje. En la práctica, cuando el psicólogo informa sobre algún resultado de una prueba, solo se enfocamos en el puntaje final de dicha prueba, dejando de lado la distribución de las respuestas correctas e incorrectas en el desempeño del evaluado. Esta aspecto psicométrico es importante porque dos puntajes iguales pueden derivarse de diferentes patrones de respuesta (PR). En la situación ideal, un patrón de respuesta esperado es aquel que los ítems más difíciles son respondidos exitosamente por sujetos con alto nivel en el atributo, mientras que los sujetos con bajo nivel en el atributo no podrían responderlos. Cuando el patrón de respuesta no se ajusta al patrón esperado, ocurre un patrón de respuesta atípico (PRA); en esta situación, los sujetos más hábiles fallan en las tareas fáciles; y los sujetos menos hábiles aciertan en las tareas difíciles. Por tal motivo nuestra investigación tiene por objetivos 1) Determinar la frecuencia ocurre el patrón de respuesta atípico, y 2) su variación respecto al tipo de prueba, y al tiempo y lugar de recolección de datos. La muestra de 174 niños preescolares ingresantes al primer grado de primaria, provenientes de dos colegios públicos en una zona urbana, de un distrito costero al sur de Lima Metropolitana. Todos los niños fuero de nivel socioeconómico medio bajo o menor. El instrumento aplicado fue una batería de pruebas para detección de problemas en habilidades pre-académicas, pero se usaron específicamente las subescalas Conocimiento de Letras y Palabras, Conceptos Cuantitativos, y Vocabulario/Conceptos. La prueba se administró grupalmente, independientemente en cada colegio;

${ }^{\star}$ Trabajo presentado en el Encuentro Científico Internacional - Verano 2009, realizado el 6 - 9 de Enero del 2009, en la Universidad Tecnológica del Perú, Lima, Perú.

$\star \star$ Estudiantes en la Escuela Profesional de Psicología, Universidad Nacional Federico Villareal, Lima, Perú.

$\star \star \star$ Psicólogo, maestría en psicología educativa. Actualmente investigador y docente del Instituto de Investigación en Psicología (Universidad de San Martín de Porres), así como en la Universidad Nacional Federico Villareal. Dirección: Calle Filiberto Romero 430, Chorrillos - Lima 9, Perú. E-mail: sikayax@yahoo.com.ar 
y en dos grupos independientes de niños. Para el análisis se aplicó un enfoque no paramétrico, usando el Indice Modificado de Precaución (IMP, Harnisch \& Linn, 1981; Sato, 1986) para la detección de PRA; el criterio fue IMP $>0.30$. Nuestros resultados indican, en primer lugar, la prevalencia de un porcentaje entre $0.30 \%$ y $50 \%$ de puntajes con PRA; esta frecuencia es relativamente elevado para fines profesionales, y se pueden interpretar como poco válidos. En segundo lugar, nuestros resultados demuestran una aparente relación entre el tipo de prueba y la frecuencia del PRA, y que la prevalencia parece ser constante en los dos colegios muestreados en dos años diferente. Se discute el impacto de estilos de respuesta particulares, el trasfondo cultural atípico, o condiciones de evaluación variables; así mismo, planteamos que el PRA puede ser prevalerte en la evaluación del rendimiento escolar y contener un componente inválido, o aleatorio. 